\title{
Pulmonary Contusion in Mechanically Ventilated Subjects After Severe Trauma
}

\author{
Sakshi Mathur Dhar MD, Matthew D Breite MD, Stephen L Barnes MD, and Jacob A Quick MD
}

BACKGROUND: Pulmonary contusions are thought to worsen outcomes. We aimed to evaluate the effects of pulmonary contusion on mechanically ventilated trauma subjects with severe thoracic injuries and hypothesized that contusion would not increase morbidity. METHODS: We conducted a single-center, retrospective review of $\mathbf{1 6 3}$ severely injured trauma subjects (injury severity score $\geq$ 15) with severe thoracic injury (chest abbreviated injury score $\geq 3$ ), who required mechanical ventilation for $>\mathbf{2 4} \mathrm{h}$ at a verified Level 1 trauma center. Subject data were analyzed for those with radiographic documentation of pulmonary contusion and those without. Statistical analysis was performed to determine the effects of coexisting pulmonary contusion in severe thoracic trauma. RESULTS: Pulmonary contusion was present in 91 subjects $(55.8 \%)$, whereas $72(44.2 \%)$ did not have pulmonary contusions. Mean chest abbreviated injury score $(3.54$ vs $3.47, P=.53)$ and mean injury severity score $(32.6 \mathrm{vs} 30.2, P=.12)$ were similar. There was no difference in mortality (11 $[12.1 \%]$ vs $9[12.5 \%], P>.99)$ or length of stay $(16.29 \mathrm{~d}$ vs $17.29 \mathrm{~d}, P=.60)$. Frequency of ventilator-associated pneumonia was comparable $(43[47.3 \%]$ vs $32[44.4 \%], P=.75)$. Subjects with contusions were more likely to grow methicillin-sensitive Staphylococcus aureus in culture (33 vs 10, $P=.004)$ as opposed to Pseudomonas aeruginosa in culture (6 vs $13, P=.003)$. CONCLUSIONS: Overall, no significant differences were noted in mortality, length of stay, or pneumonia rates between severely injured trauma subjects with and without pulmonary contusions. Key words: ventilator-associated pneumonia; trauma; pulmonary contusion; outcomes; thoracic; ARDS. [Respir Care 2018;63(8):950-954. (C) 2018 Daedalus Enterprises]

\section{Introduction}

Pulmonary contusions range from mild to life-threatening injury. Commonly diagnosed in patients who suffer blunt chest trauma, the incidence in these populations reaches $65 \%$. Pediatric patients have an even higher rate of significant lung injury of up to $80 \%$, secondary to increased chestwall compliance. ${ }^{1}$ Isolated pulmonary contusion is rare, and often the rate of pulmonary contusion is linearly associated with severity of injury to the bony tho-

\footnotetext{
The authors are affiliated with the Division of Acute Care Surgery, Department of Surgery, University of Missouri, Columbia, Missouri.

The authors have disclosed no conflicts of interest.

Correspondence: Jacob A Quick MD, University of Missouri Department of Surgery, Division of Acute Care Surgery, 1 Hospital Drive, MC220, Columbia, MO 65212. E-mail: quickja@ health.missouri.edu.
}

DOI: $10.4187 /$ respcare. 05952 rax. Rib fractures and their degree of displacement, as well as flail chest and penetrating mechanisms, all contribute to severity of the underlying lung injury. ${ }^{1-3}$

Shear forces associated with the development of pulmonary contusions cause several local and systemic effects. Mucus production is increased. Oxygen radicals and cytokines are released. Protein accumulates, and surfactant production is reduced. The end result is atelectasis and consolidation. These mechanical and inflammatory sequelae have the propensity to complicate care of those with severe chestwall injury, potentially increasing the risk of pneumonia, acute respiratory failure, and ARDS.4-6 The morbid sequelae of pulmonary contusion are most often seen in those who have failed conservative therapy and require mechanical ventilation to improve gas exchange and prevent hypoxemia., ${ }^{4,6}$ Mechanically ventilated patients are also at increased risk to develop ventilator-associated events, such as barotrauma, volutrauma, and infectious complications. ${ }^{4,8,9}$

Prior studies have failed to isolate pulmonary contusion as the causative agent of worsened outcomes among those 


\section{Pulmonary Contusion in Mechanically Ventilated Subjects}

with severe injuries because of the prevalence of concurrent traumatic injury. ${ }^{10}$ With severe thoracic injury, it is difficult to discern whether the unwanted sequelae are secondary to thoracic injury or to the underlying lung injury

\section{See the Related Editorial on Page 1073}

specifically. We sought to evaluate the outcomes of mechanically ventilated patients with severe thoracic injuries, with and without pulmonary contusion, and hypothesized that contusion would not increase morbidity.

\section{Methods}

A retrospective cohort study was undertaken at our institution, an American College of Surgeons Level 1 trauma center, following institutional review board approval. All consecutive trauma patients admitted over a 30-month period who required mechanical ventilation for $>24 \mathrm{~h}$ were evaluated. To ensure that severe injury was present, patients with a chest abbreviated injury score $<3$ and injury severity score $<15$ were excluded. The remaining subjects were then divided into 2 groups for analysis: (1) those with documented plain chest radiograph evidence of pulmonary contusion within $24 \mathrm{~h}$ of admission and (2) those without radiographic evidence of pulmonary contusion. We did not include patients with evidence of pulmonary contusion only seen on computed tomography examinations, as these injuries are typically not clinically important. ${ }^{11,12}$ To obtain the radiographic diagnosis of contusion and decrease bias, all radiographs were interpreted by a resident radiologist, faculty radiologist, and blinded attending acutecare surgeon. Radiographic diagnosis was only obtained when all 3 evaluators were in agreement. Demographics, types of injury, length of hospital stay, operative data, comorbidities, intubation data, microbiologic data, imaging, and laboratory data were analyzed. Ventilator-associated pneumonia (VAP) was diagnosed utilizing clinical and microbiologic criteria. Clinical VAP diagnosis required at least 3 of the following: new or progressive infiltrate on chest radiograph; fever $>38.5^{\circ} \mathrm{C}$; leukocytosis $(>12,000)$ or leukopenia $(<4,000)$; purulent tracheal secretions; and absence of post-traumatic or postsurgical causes for clinical findings. If clinical criteria were met, diagnosis was confirmed microbiologically with $>10^{4}$ colony-forming units of a single predominant organism obtained via bronchoalveolar lavage. Statistical analysis was performed using 2-tailed Student $t$ test, Fisher exact test, and chi-square formulas. Statistical significance was set to $95 \%(P<.05)$.

\section{QUICK LOOK}

\section{Current knowledge}

Severe thoracic injury is often associated with pulmonary contusions. Direct pulmonary parenchymal injury can result in worsened overall lung function and create difficulties in management. In the trauma setting, the presence of pulmonary contusions is thought to increase the morbidity and mortality of injured patients.

\section{What this paper contributes to our knowledge}

To identify the effect of pulmonary contusion on patients with severe thoracic injury, we reviewed only subjects who required mechanical ventilation, who also had elevated injury scores. Whereas we identified the potential to require advanced ventilator management in the setting of pulmonary contusions, there appeared to be no difference in morbidity, length of stay, or mortality between subjects suffering pulmonary contusion and those without.

\section{Results}

During the study period, 324 patients were admitted to the trauma ICU and required mechanical ventilation for $>24 \mathrm{~h}$. After applying exclusion criteria, 163 subjects remained for analysis. Pulmonary contusion was present in 91 subjects $(55.8 \%)$, whereas 72 subjects $(44.2 \%)$ did not suffer pulmonary contusion. Mean ages were similar (44 y vs $50 \mathrm{y}, P=.10)$, and a similar percentage of subjects were male (66 [73\%] vs 52 [72\%], $P>.99$ ) in each group. There were no differences between preexisting comorbid status or smoking history between groups. Mean body mass indices $\left(29.3 \mathrm{~kg} / \mathrm{m}^{2}\right.$ vs $\left.32.5 \mathrm{~kg} / \mathrm{m}^{2}, P=.24\right)$ were similar for those with contusion and those without (Table 1). Mean chest abbreviated injury score (3.54 vs 3.47 , $P=.53)$ and mean injury severity score (32.6 vs 30.2 , $P=.12$ ) were similar between the 2 groups. All other abbreviated injury score areas did not statistically differ between groups, except for the abdomen abbreviated injury score ( 1.8 vs $1.1, P<.003,95 \% \mathrm{CI}-1.23$ to -0.25 ), which was higher in subjects with pulmonary contusion. Hospital length of stay ( $16.3 \mathrm{~d}$ vs $17.3 \mathrm{~d}, P=.60$ ) was similar between groups. There was no difference in mortality $(11[12.1 \%]$ vs $9[12.5 \%], P>.99)$ or discharge disposition.

Surgical procedures, both in mean total number of operations (2.11 vs $2.1, P=.89)$ and type of operation, between the groups were not significantly different. Tracheostomy rates were not statistically significant $(41 \%$ vs $46 \%, P=.33$ ), nor was the duration of intubation with 
Table 1. Comorbid Status of Subjects Without and With Pulmonary Contusion

\begin{tabular}{lccc}
\hline \hline \multicolumn{1}{c}{ Characteristics } & $\begin{array}{c}\text { No } \\
\text { Contusion } n(\%)\end{array}$ & $\begin{array}{c}\text { Contusion } \\
n(\%)\end{array}$ & $P$ \\
\hline Hypertension & $24(33)$ & $22(24)$ & .22 \\
Diuretic use & $17(24)$ & $11(12)$ & .062 \\
Cardiac disease & $8(11)$ & $9(10)$ & .80 \\
Hyperlipidemia & $13(18)$ & $11(12)$ & .37 \\
Hepatic dysfunction & $2(3)$ & $1(1)$ & .58 \\
Chronic kidney disease & $2(3)$ & $1(1)$ & .58 \\
COPD or asthma & $5(7)$ & $4(4)$ & .51 \\
Smoking & $23(32)$ & $27(30)$ & .86 \\
Diabetes type 1 or 2 & $12(17)$ & $14(15)$ & .83 \\
Mean BMI, kg/m & \\
& 32.5 & 29.3 & .24 \\
BMI = body mass index & & & \\
& & & \\
\hline
\end{tabular}

mechanical ventilation before tracheostomy ( $6.5 \mathrm{~d}$ vs $7.6 \mathrm{~d}$, $P=.32$ ). Mean total blood product transfusion amounts $(1,153 \mathrm{~mL}$ vs $827 \mathrm{~mL}, P=.20)$ as well as daily fluid balances $(+669 \mathrm{~mL}$ vs $+627 \mathrm{~mL}, P=.69)$ were similar between those with contusion and those without. Mean vital signs and laboratory results were similar between groups, with the exception of statistically higher mean blood pressure seen in the contusion group (Table 2).

Regarding ventilator data, there were no significant differences seen in mean duration of mechanical ventilation per subject ( $10.5 \mathrm{~d}$ vs $11.0 \mathrm{~d}, P=.68$ ). Similar mean $\mathrm{F}_{\mathrm{IO}_{2}}$ was seen in each group (0.47 vs $0.47, P=.94)$. The number of days requiring $\mathrm{F}_{\mathrm{IO}_{2}}>0.6$ was similar as well (158 d [16.7\%] vs $136 \mathrm{~d}$ [16.8\%], $P>.99$ ). No differences in mean airway pressure $\left(13.5 \mathrm{~cm} \mathrm{H}_{2} \mathrm{O}\right.$ vs $13.7 \mathrm{~cm} \mathrm{H}_{2} \mathrm{O}$, $P=.21)$ or peak airway pressure $\left(24.8 \mathrm{~cm} \mathrm{H}_{2} \mathrm{O}\right.$ vs $27.1 \mathrm{~cm} \mathrm{H}_{2} \mathrm{O}, P=.16$ ) were observed. Subjects with pulmonary contusion had statistically higher tidal volumes with respect to predicted body weight $(7.52 \mathrm{~mL} / \mathrm{kg}$ vs $7.18 \mathrm{~mL} / \mathrm{kg}, P=.053,95 \% \mathrm{CI}-0.006$ to 0.69$)$ and statistically higher set ventilator inspiratory pressures to reach those tidal volumes $\left(14.9 \mathrm{~cm} \mathrm{H}_{2} \mathrm{O}\right.$ vs $14.5 \mathrm{~cm} \mathrm{H}_{2} \mathrm{O}$, $P=.040,95 \%$ CI -0.88 to -0.02$)$. Mean PEEP was statistically higher in the group without contusion (9.2 $\mathrm{cm} \mathrm{H}_{2} \mathrm{O}$ vs $9.5 \mathrm{~cm} \mathrm{H}_{2} \mathrm{O}, P=.033$, 95\% CI $0.02-$ $0.61)$. Most subjects in both groups were ventilated using pressure control ventilation. However, more subjects with pulmonary contusion were ventilated with airway pressure release ventilation (APRV) $(39$ [42.9\%] vs 1 [1.3\%], $P=$ $.001)$.

Ventilator-associated events, specifically VAP, occurred with similar frequency in both groups (43 [47.3\%] vs 32 [44.4\%], $P=.75)$. Subjects in the contusion group who were diagnosed with pneumonia had 134 positive cultures, whereas subjects without contusion had 102 positive cultures. Both groups had similar numbers of positive cul-
Table 2. Mean Vital Signs and Laboratory Results During ICU Length of Stay

\begin{tabular}{lrrc}
\hline \hline \multicolumn{1}{c}{ Characteristics } & No Contusion & Contusion & $P$ \\
\hline $\mathrm{WBC}, 10^{9} / \mathrm{L}$ & 12.87 & 13.94 & $<.001$ \\
Hemoglobin, g/dL & 9.86 & 9.72 & .33 \\
Platelets, $10^{9} / \mathrm{L}$ & 285.67 & 289.80 & .65 \\
$\mathrm{INR}$ & 1.26 & 1.23 & .35 \\
Fibrinogen, g/L & 326.49 & 332.69 & .81 \\
$\mathrm{HCO}_{3}\left(\mathrm{CO}_{2}\right), \mathrm{mEq} / \mathrm{L}$ & 26.16 & 25.92 & .17 \\
$\mathrm{~S}_{\overline{\mathrm{cv}}}$ & 70.49 & 73.06 & .13 \\
$\mathrm{pH}$ & 7.40 & 7.40 & .87 \\
$\mathrm{P}_{\mathrm{CO}_{2}}$ & 38.79 & 39.27 & .35 \\
$\mathrm{P}_{\mathrm{O}_{2}}$ & 120.16 & 114.03 & .18 \\
$\mathrm{Base}_{2}$ excess & -0.58 & -0.17 & .11 \\
$\mathrm{~T}_{\mathrm{max}}$, degrees C & 37.67 & 37.62 & .21 \\
$\mathrm{HR}_{\text {, beats/min }}$ & 96.62 & 96.49 & .87 \\
Frequency, breaths/min & 20.71 & 20.18 & .10 \\
$\mathrm{SBP}$, mm Hg & 124.80 & 128.91 & $<.001$ \\
$\mathrm{DBP}, \mathrm{mm} \mathrm{Hg}$ & 67.89 & 69.98 & .002 \\
$\mathrm{~S}_{\mathrm{pO}_{2}}, \%$ & 96.81 & 96.75 & .60
\end{tabular}

Data are presented as the mean value for the ICU length of stay (d).

$\mathrm{WBC}=$ white blood cell

$\mathrm{INR}=$ international normalized ratio

$\mathrm{HCO}_{3}=$ serum bicarbonate

$\mathrm{S}_{\overline{\mathrm{cv}} \mathrm{O} 2}=$ central mixed venous oxygen

$\mathrm{T}_{\max }=$ maximum daily recorded temperature

$\mathrm{HR}=$ heart rate

$\mathrm{SBP}=$ systolic blood pressure

$\mathrm{DBP}=$ diastolic blood pressure

tures per subject (3.1/subject vs 3.2/subject, $P=.79)$. Significant differences were noted in the most common offending organisms causing VAP. Subjects with pulmonary contusion were more likely to grow methicillin-sensitive Staphylococcus aureus in culture (33 vs 10, odds ratio 3.0, $P=.004,95 \%$ CI 1.4-6.4). Conversely, subjects without pulmonary contusion were more likely to grow Pseudomonas aeruginosa in culture (6 vs 13, odds ratio $0.32, P=.029,95 \% \mathrm{CI} 0.11-0.88)$. Both groups commonly grew Haemophilus influenzae (13 vs 19, $P=.08)$, Enterobacter species (15 vs $10, P=.73)$ and Candida species (10 vs $8, P=.91)$ (Table 3).

\section{Discussion}

Overall, our study showed few significant differences between subjects who sustained pulmonary contusions and subjects who did not, after significant chest trauma. Mortality rates were similar. There was no effect on the stay or on the discharge disposition between the 2 groups. Both groups experienced similar mechanical ventilation duration, without significant differences in rates of tracheostomy or development of VAP. No differences were seen in laboratory results or vital signs between those with pulmonary contusion and those without. 
Table 3. Culture Data

\begin{tabular}{|c|c|c|c|c|}
\hline \multirow{2}{*}{ Culture } & \multicolumn{2}{|c|}{$\begin{array}{c}\text { Number of } \\
\text { Positive Cultures }\end{array}$} & \multirow{2}{*}{$\begin{array}{l}\text { Odds } \\
\text { Ratio }\end{array}$} & \\
\hline & $\begin{array}{c}\text { No } \\
\text { Contusion }\end{array}$ & Contusion & & \\
\hline MSSA & 10 & 33 & 3.0 & .004 \\
\hline Hemophilus influenzae & 13 & 19 & 1.13 & .08 \\
\hline Enterobacter species & 10 & 15 & 1.16 & .73 \\
\hline Acinetobacter species & 5 & 10 & 1.56 & .43 \\
\hline Candida species & 8 & 10 & 0.95 & .91 \\
\hline Escherichia coli & 5 & 7 & 1.07 & .91 \\
\hline Streptococcus pneumoniae & 6 & 6 & 0.75 & .63 \\
\hline Pseudomonas species & 13 & 6 & 0.32 & .03 \\
\hline MRSA & 9 & 5 & 0.40 & .11 \\
\hline Serratia species & 8 & 0 & 0.04 & .03 \\
\hline \multicolumn{5}{|c|}{$\begin{array}{l}\text { Odds are ratios expressed as odds with pulmonary contusion. } \\
\text { MSSA }=\text { methicillin-sensitive Staphylococcus aureus } \\
\text { MRSA = methicillin-resistant } S \text {. aureus }\end{array}$} \\
\hline
\end{tabular}

Whereas other studies have described the association between pulmonary contusions and VAP, this study is particularly salient due to the methods used to differentiate between thoracic injury and thoracic injury with pulmonary contusion. Inclusion in the study required subjects to have a chest abbreviated injury score $\geq 3$ and an injury severity score $\geq 15$. This stratified our subjects to select only the most severely injured. Our comparison could then be conducted between 2 groups with the only difference being contusion or lack thereof. Despite the retrospective design of the study, the 2 groups were statistically identical with regard to demographics and premorbid conditions, decreasing the effect of confounding variables. This allowed us to successfully differentiate between the effects of severe thoracic injury and those of pulmonary contusion.

Previous studies have shown that mean hospital and ICU lengths of stay increase with the presence of pulmonary contusions, probably because subjects with contusion also had serious chestwall injury, whereas those without contusion had minimal chestwall injury. ${ }^{5,8,13,14}$ Thus, data were skewed to evaluate the severity of injury rather than the contributory effect of pulmonary contusion. Our study, which compares subjects with similarly severe injuries, found no difference in length of stay between those who sustained pulmonary contusion and those who did not, despite severe thoracic trauma. Similarly, and contrary to previously published data, we identified no statistical differences in mortality rates between subjects with pulmonary contusion and those without. ${ }^{11,15}$

Pulmonary contusions have been associated with longer duration of mechanical ventilation, as well as higher PEEP and oxygen requirements. ${ }^{13,16,17}$ Contrary to other studies, we saw similar duration of mechanical ventilation, oxygen requirements, and rates of tracheostomy between the 2 groups studied. This can be explained by both groups suffering similar degrees of thoracic injury and supports the lack of difference in outcomes when pulmonary contusions are coincident. When selecting a ventilation mode, the physiologic state of the lung parenchyma is considered. Settings are customized to prevent ventilation-induced lung injury. In our study, pressure control ventilation was most commonly employed in subjects with contusion as well as those without contusion. Pressure support was the second most common mode utilized in both groups. With more advanced modes of ventilation, we did see a notable difference between the studied groups. APRV was more often utilized in subjects with pulmonary contusion. The significance of this is undetermined, but it may represent the need for higher mean airway pressures and additional lung recruitment when contusions are present, thus favoring APRV. Our initial settings for APRV are $\mathrm{T}_{\text {high }}=4.5 \mathrm{~s}, \mathrm{~T}_{\text {low }}=0.5 \mathrm{~s}, \mathrm{P}_{\text {low }}=0$, and $\mathrm{P}_{\text {high }}=30$, which aids in recruitment. Settings are adjusted to the degree that permissive hypercapnia may be tolerated and are patientdependent. The use of APRV to improve recruitment and oxygenation may have positively affected survival, and thus normalized mortality rates, although the study was not designed to detect these suggested mechanisms.

Elevated pneumonia risk has been well documented in patients requiring mechanical ventilation, and multiple studies have outlined this increased risk in subjects who suffered significant trauma. ${ }^{10,17-20}$ Additionally, higher VAP rates have been associated with the presence of pulmonary contusions. ${ }^{19,21}$ We saw a relatively high VAP rate in both groups of our study. This is probably reflected by the severity of chest injury as well as the overall injury severity reflected by our inclusion criteria. However, our data showed no difference in VAP rates between subjects with and without pulmonary contusion. Previous authors have described increasing pulmonary risks with the degree of pulmonary contusion. ${ }^{16}$ We did not quantify contusion volumes or grade contusion severity, which may have contributed to the lack of difference in VAP rates seen in this study. However, our data suggest that the mere existence of contusion in the setting of severe thoracic trauma does not affect pneumonia rates.

Ventilator-associated pneumonia is commonly due to organisms such as methicillin-resistant S. aureus, Pseudomonas, Klebsiella, Escherichia coli, Acinetobacter, and Enterobacter. ${ }^{22}$ Our data showed many of these known common causes of VAP to be present in both groups, including $H$. influenzae, Enterboacter species, and Candida species. Interestingly, we saw marked differences between the 2 most commonly identified pathogens in this study. Contused subjects were most likely to grow methicillin-sensitive $S$. aureus in culture, with 3 times the number of methicillin-sensitive $S$. aureus cases seen in subjects with contusion. Conversely, subjects without lung contusion were more 
likely to grow $P$. aeruginosa or Serratia species, which are typically regarded as organisms with relatively high virulence and pathogenicity. This is somewhat counterintuitive, as one would expect more severely injured lung parenchyma would be more susceptible to more virulent organisms. However, this was not the case in our study. It may be that severe parenchymal injury makes the lung more susceptible to consolidation early, leading to earlier infection with less virulence, whereas patients with less direct lung injury are more susceptible later in the hospital stay, and thus more susceptible to infection from more virulent causes, due to prolonged exposure in the ICU.

Our study was limited by the constraints of a retrospective review at a single institution. Our sample size was thus relatively small, and ventilator settings were not randomized. Due to the strict inclusion criteria that selected for only those subjects with severe thoracic injury, our sample size was underpowered to detect small differences in mortality and morbidity related to pulmonary contusion. Additionally, interpretation of radiographs is inherently subjective. We aimed to control for this via required redundancy and inter-observer agreement in regard to radiographic interpretation; however, bias may have affected results.

\section{Conclusions}

We found no significant differences in outcomes between groups with and without pulmonary contusions. Mortality and length of stay were similar. Ventilator days, tracheostomy rates, and duration of mechanical ventilation were also similar. In severely injured subjects, pulmonary contusions did not appear to play a role in outcomes.

\section{REFERENCES}

1. Balci AE, Kazez A, Eren S, Ayan E, Ozalp K, Eren MN. Blunt thoracic trauma in children: review of 137 cases. Eur J Cardiothorac Surg 2004;26(2):387-392.

2. Skinner DL, den Hollander D, Laing GL, Rodseth RN, Muckart DJ. Severe blunt thoracic trauma: differences between adults and children in a level I trauma centre. S Afr Med J 2015;105(1):47-51.

3. Hoessini M, Ghelichkhani P, Baikpour M, Tafakhori A, Asady H, Haji Ghanbari MJ, et al. Diagnostic accuracy of ultrasonography and radiography in detection of pulmonary contusion; a systemic review and meta-analysis. Emerg 2015;3(4):127-136.

4. Cohn SM, Dubose JJ. Pulmonary contusion: an update on recent advances in clinical management. World J Surg 2010;34(8):1959-1970.

5. Alisha C, Gajanan G, Jyothi H. Risk factors affecting the prognosis in patients with pulmonary contusion following chest trauma. J Clin Diagn Res 2015;9(8):OC17-OC19.
6. Ganie FA, Lone H, Lone GN, Wani ML, Singh S, Dar AM, et al. Lung contusion: a clinico-pathological entity with unpredictable clinical course. Bull Emerg Trauma 2013;1(1):7-16.

7. Simon BJ, Cushman J, Barraco R, Lane V, Luchette FA, Miglietta $\mathrm{M}$, et al. Pain management guidelines for blunt thoracic trauma. J Trauma 2005;59(5):1256-1267.

8. Simon B, Ebert J, Bokhari F, Capella J, Emhoff T, Hayward T 3rd, et al. Management of pulmonary contusion and flail chest: an Eastern Association for the Surgery of Trauma practice management guideline. J Trauma Acute Care Surg 2012;73(5 Suppl 4):S351-S361.

9. Cabrera-Benitez NE, Laffey JG, Parotto M, Spieth PM, Villar J, Zhang H, Slutsky AS. Mechanical ventilation-associated lung fibrosis in acute respiratory distress syndrome: a significant contributor to poor outcome. Anesthesiology 2014;121(1):189-198.

10. Landeen C, Smith HL. Examination of pneumonia risks and risk levels in trauma patients with pulmonary contusion. J Trauma Nurs 2014;21(2):41-49.

11. Rodriguez RM, Friedman B, Langdorf MI, Baumann BM, Nishijima DK, Hendey GW, et al. Pulmonary contusion in the pan-scan era. Injury 2016;47(5):1031-1034.

12. Kea B, Gamarallage R, Vairamuthu H, Fortman J, Lunney K, Hendey GW, Rodriguez RM. What is the clinical significance of chest $\mathrm{CT}$ when the chest $\mathrm{X}$-ray result is normal in patients with blunt trauma? Am J Emerg Med 2013;31(8):1268-1273.

13. Clark GC, Schecter WP, Trunkey DD. Variables affecting outcome in blunt chest trauma: flail chest vs. pulmonary contusion. J Trauma 1988;28(3):298-304.

14. Flagel BT, Luchette FA, Reed RL, Esposito TJ, Davis KA, Santaniello JM, Gamelli RL. Half-a-dozen ribs: the breakpoint for mortality. Surgery 2005;138(4):717-723; discussion 723-725.

15. Balci AE, Balci TA, Eren S, Ulkü R, Cakir O, Eren N. Unilateral post-traumatic pulmonary contusion: findings of a review. Surg Today 2005;35(3):205-210.

16. Miller PR, Croce MA, Bee TK, Qaisi WG, Smith CP, Collins GL, Fabian TC. ARDS after pulmonary contusion: accurate measurement of contusion volume identifies high risk-patients. J Trauma 2001; 51(2):223-228; discussion 229-230.

17. Mangram AJ, Sohn J, Zhou N, Hollingworth AK, Ali-Osman FR, Sucher JF, et al. Trauma-associated pneumonia: time to redefine ventilator-associated pneumonia in trauma patients. Am J Surg 2015; 210(6):1056-1061; discussion 1061-1062.

18. Richardson JD, Adams L, Flint LM. Selective management of flail chest and pulmonary contusion. Ann Surg 1982;196(4):481-487.

19. Antonelli M, Moro ML, Capelli O, De Blasi RA, D'Errico RR, Conti $\mathrm{G}$, et al. Risk factors for early onset pneumonia in trauma patients. Chest 1994;105(1):224-228.

20. Magret M, Amaya-Villar R, Garnacho J, Lisboa T, Díaz E, Dewaele $\mathrm{J}$, et al. Ventilator-associated pneumonia in trauma patients is associated with lower mortality: results from EU-VAP study. J Trauma 2010;69(4):849-854.

21. Walkey AJ, Nair S, Papadopoulos S, Agarwal S, Reardon CC. Use of airway pressure release ventilation is associated with a reduced incidence of ventilator-associated pneumonia in patients with pulmonary contusion. J Trauma 2011;70(3):E42-E47.

22. Rumbak MJ. Pneumonia in patients who require prolonged mechanical ventilation. Microbes Infect 2005;7(2):275-278.

This article is approved for Continuing Respiratory Care Education credit. For information and to obtain your CRCE

(free to AARC members) visit www.rcjournal.com

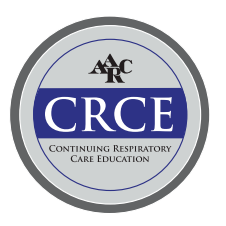

